\title{
SOBRE LA TRADUCCIÓN LITERARIA Y LA IDENTIDAD DEL TRADUCTOR
}

\author{
MaARTen SteenmeiJer \\ Radboud Universiteit Nijmegen \\ m.steenmeijer@let.ru.nl
}

RESUMEN: Es una idea generalizada que la traducción literaria es, por definición, una pobre versión del original. Por otra parte, sigue vigente la idea de que la equivalencia es el criterio decisivo a la hora de evaluar y juzgar las traducciones literarias. Este artículo defiende la tesis de que la equivalencia es una falacia y propone otro enfoque. Traducir es, en realidad, escribir. O para ser más específico: traducir es escribir con dos voces, la del escritor y la del mismo traductor. Así, el traductor finge la voz del escritor y miente el original, convirtiendo la traducción literaria en el discurso literario por antonomasia: así como el texto original, la traducción literaria finge la realidad pero, además, finge el original.

PALABRAS CLAVE: traducción literaria; equivalencia; voz del traductor; identidad de la traducción literaria

ABSTRACT: It is widely believed that a literary translation is by definition a poor version of the original. On the other hand, the idea that equivalence is the decisive criterion when evaluating and judging literary translations is also very persistent. This article defends the thesis that equivalence is a fallacy and proposes another perspective. Translation is, actually, writing. Or to be more specific: translation is writing with two voices, the writer's voice and the translator's own 
voice. In fact, the translator belies the original, making literary translations the literary discourse par excellence: besides belying reality, just like every literary text does, a literary translation belies the original text.

KEYWORDS: Literary Translation; Equivalence; Translator's Voice; Literary Translation's Identity

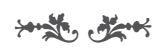

En Apocryphal Lorca. Translation, parody, kitsch (2009) Jonathan Mayhew investiga la traducción y recepción de la obra de Federico García Lorca en los Estados Unidos en los años 50 y 60 del siglo pasado. Es el periodo en que culminó el impacto del escritor granadino, que, junto con Rainer Maria Rilke, es el poeta europeo que más huellas dejó en la poesía norteamericana del siglo xx. Mayhew revela que la acogida de la poesía de García Lorca fue polifacética y que servía de antídoto contra la epidemia anticomunista de McCarthy que por aquel entonces azotaba a los Estados Unidos (Mayhew 2009: 27).

Sin embargo, por diversa y multicultural que fuera la recepción del poeta granadino en los EE.UU, Mayhew no vacila en deplorar y reprobarla. Es más: según el estudioso norteamericano, este país no acertó a apreciar debidamente la obra de García Lorca, como ya sugiere el título de su libro, que incluye palabras tendenciosas y estigmatizantes como "apócrifo", "kitsch" y "parodia". Para empezar, Mayhew juzga problemáticas las dos traducciones con las que García Lorca llegó a la fama en los EE.UU. La primera es Selected Poems, una antología de la poesía de Lorca que desde su publicación en 1955 ya lleva vendidos más de 120.000 ejemplares. La otra es The Poet in New York, que incluye, aparte de una versión inglesa del poemario Poeta en Nueva York, una traducción de la conferencia "Juego y teoría del duende". Mayhew rechaza los dos libros en su capacidad de traducción: el primero por no hacer justicia al tono y las metáforas del original y el segundo porque el poeta que lo tradujo, Ben Belitt, habría canibalizado el texto original en beneficio de su propio proyecto poético.

Concluye Mayhew que, por variada que haya sido la acogida del poeta español, el Lorca norteamericano plasmado en las traducciones y adaptaciones de su poesía y en los poemas escritos por poetas norteamericanos sobre o con motivo de la obra y figura del poeta andaluz es, en el fondo, un pequeño repertorio de clichés sobre España (cuya cultura se caracterizaría por ser profunda, auténtica, irracional, intuitiva, inspirada y surrealista) y unos cuantos temas e imágenes (caballos, luna, gitanos, duende, Nueva York, Walt Whitman). Asevera el autor de Apocryphal Lorca que esta apropiación o usurpación no hace justicia al Lorca "real" (una palabra que, sorprendentemente, Mayhew maneja sin reserva alguna), un autor mucho más complejo o, en las palabras de Mayhew, "a self-aware artist with a deep and nuanced understanding of the Spanish literary tradition" (9) y "a cosmopolitan intellectual who came of age during the epoch of the historical avant-garde in Europe" (10). Con todo, Mayhew concluye que en los Estados Unidos el poeta español se convirtió en un poeta norteamericano y, 
para más inri del estudioso, en un poeta mediocre, cuya pluralidad y complejidad no fueron reconocidas correctamente.

La implacable crítica de Mayhem forma parte de un largo y pertinaz paradigma según el cual la traducción y, en particular, la traducción literaria es, por definición, una sombra del original o incluso una falsa versión de este. Según este mismo paradigma, el destino prototípico de un texto vertido a otro idioma sería el desarraigo. Incluso los mismos traductores viven el exilio en su propio idioma, como afirma Suzanne Jill Levine en The Subversive Scribe (1991: 1). De este modo, la traducción literaria goza de una reputación poco envidiable, como atestiguan los lugares comunes sobre la traducción literaria, repetidos hasta la saciedad. Así, reza el adagio italiano paranomásico que el traductor es un traidor ("traduttore, traditore") mientras que, según la expresión francesa no exenta de sexismo "belles infidèles", las traducciones estéticamente satisfactorias no pueden ser fieles a los textos originales. Es más: según Sylvia Durastanti, el traductor sería un agente doble: "Il faut bien dire qu'il n'est de traduction réussie que par une double trahison: celle de la langue prétendument maternelle du traducteur et celle de l'autre, prétendument étrangère" (Durastanti 2002: 9). También vale la pena recordar aquí la muy citada afirmación atribuida al poeta norteamericano Robert Frost de que la poesía -que no sería exagerado calificar como la forma más condensada e intrincada de la literatura- es lo que se pierde en la traducción ("Poetry is what gets lost in translation"), una idea que Roman Jakobson defiende y elabora en su clásico ensayo "Sobre los aspectos lingüísticos de la traducción", de 1959.

La idea de que en la traducción literaria se borra, se esfuma o se diluye lo propio o lo otro del texto original es, asimismo, la idea que guía The Translator's Invisibility (1995), el acreditado libro en que Lawrence Venuti condena la estrategia domesticadora que, según el teórico y traductor norteamericano, caracteriza la traducción literaria en el mundo anglosajón. Vale la pena, asimismo, recordar aquí el determinismo de Schopenhauer expresado en "Sobre lengua y palabras":

Casi nunca puede trasladarse la cláusula característica, concisa y significativa de una lengua a otra de forma tal que surta exacta y completamente idéntico efecto. [...] Por esta razón, toda traducción es una traducción muerta, y su estilo será forzado, formal, artificial: o, en caso contrario, será una traducción libre, es decir, se limitará a un à peu près, y por lo tanto será falsa. Una biblioteca de traducciones es como una galería de reproducciones de pinturas. Por no hablar de las traducciones de los poetas de la Antigüedad, que respecto de ellos no son sino sucedáneos, en no menor medida que lo es la achicoria respecto del café. (Schopenhauer 1996: 198-199)

En esta misma línea, Eduardo Mendoza sostiene en la lúcida conferencia "La traducción y sus descontentos" que "una traducción solo puede empeorar el texto original" y que "la sensación que tiene el traductor es que su trabajo consiste simplemente en una suma de males menores y [...] en un fracaso" (Mendoza 2002). En otro pasaje de esta misma conferencia, el escritor barcelonés no vacila en especificar el tamaño del fracaso: 
Yo creo que es inevitable que en una traducción con un mínimo de dificultad, sobre todo de un texto con voluntad de ser original, de tener una cierta idiosincrasia, algunas bromas, algunos significados desplazados para provocar sorpresa en el lector, en este tipo de traducciones, hay que aceptar pérdida inevitable como de un $10 \%$, por fijar una cifra de consenso. Hay que procurar que no pase de este 10, pero al 10 hay que resignarse. (Mendoza 2002)

\section{LA EQUIVALENCIA Y LA TRADUCCIÓN IDEAL}

En su perspicaz y panorámico libro Is That a Fish in Your Ear? Translation and the Meaning of Everything (2011) el traductor, biógrafo y filólogo David Bellos sostiene categóricamente que, de hecho, no pueden ser equivalentes la traducción y el texto original:

No translation is the same as its source, and no translation can be expected to be like its source in more than a few selected ways. Which dimensions are selected depends on the conventions of the receiving culture, the nature of the field involved, or even the whim of the commissioner of the translation. (Bellos 2011: 335)

Como mucho, la equivalencia entre una traducción y el original puede darse al nivel de la palabra aunque, por poner un ejemplo aleatorio, la palabra "jazz" -en su origen una palabra inglesa que ha ido formando parte del vocabulario de muchos otros idiomas- tiene distintas connotaciones en cada uno de estos idiomas. Incluso los sonidos no solo cobran sentido en su capacidad de constituyentes de palabras, oraciones y textos, sino también por sí mismos. Así, en el inglés se asocian las oclusivas ( $p, b, t, d, k, g)$ con lo abrupto y lo autoritario, la "I" con lo ligero, la "s" con significados variados como suave, taimado y la rabia reprimida, mientras que la "o" tiene una connotación fantasmal o siniestra. Es significativo que incluso sin saber hablar ciertas lenguas extranjeras solamos asociarlas con ciertos sonidos, como se puede comprobar al escuchar a personas que imitan el chino o el ruso en su propio idioma. No es menos revelador en este contexto que, hablando a un bebé, cambiemos de tono y usemos un vocabulario muy limitado que repetimos y repetimos, suponiendo que al recién nacido, incapaz de entender estos sonidos a nivel semántico, le gustan precisamente estos mismos sonidos.

La estructura de las palabras y de los grupos de palabras varía también según el idioma. Para comprobarlo basta escuchar hablar a alguien un idioma extranjero. Sin ir más lejos, alguien que hable español y use continuamente pronombres personales como "yo", "tú", "él" y "ella" no puede ser un hispanohablante nativo, porque de lo contrario sabría por intuición que las formas verbales españolas ya suelen indicar el sujeto.

Pero en el fondo ¿no expresan los idiomas exactamente lo mismo y no son las diferencias entre unos y otros más que superficiales? ¿No percibimos todos la realidad de la misma manera? ¿O expresan las diferencias entre los idiomas también visiones divergentes sobre el mundo? ¿O incluso estructura y determina 
la lengua que hablamos nuestra manera de ver y comprender la realidad? En la lingüística hay variedad de ideas al respecto. Según el enfoque determinista, la lengua decide cómo pensamos la realidad, mientras los relativistas moderados se limitan a creer que hay, en efecto, un vínculo entre lengua y Weltanschauung. Un ejemplo revelador serían las diferencias conceptuales ligadas a los géneros gramaticales de ciertas palabras alemanas y españolas:
... the word for "key" is masculine in German and feminine in Spanish. German speakers described keys as hard, heavy, jagged, metal, serrated, and useful, whi- le Spanish speakers said they were golden, intricate, little, lovely, shiny, and tiny. The word for "bridge," on the other hand, is feminine in German and masculine in Spanish. German speakers described bridges as beautiful, elegant, fragile, peaceful, pretty, and slender, while Spanish speakers said they were big, dange- rous, long, strong, sturdy, and towering. (Boroditsky, Schmidt, Phillips 2001: 70)

Debido a las diferencias entre los idiomas hasta el nivel más elevado y más abstracto -la visión del mundo que expresan o configuran- podría decirse, efectivamente, que traducir es imposible. No puede existir una equivalencia completa entre dos textos en dos lenguas diferentes. En el fondo, una lengua solo coincide con ella misma. Podría concluirse entonces que es imposible traducir. Pero esa es una respuesta teórica. La realidad es que, lisa y llanamente, se practica la traducción.

Pueden hacerse más comentarios sobre la idea precocinada de que es imposible traducir. El primero tiene que ver con los lectores que no tengan más remedio que recurrir a una traducción por el simple hecho de que no dominan la lengua en la que está escrito el libro o de manera insuficiente para poder comprender bien un texto. Les es irrelevante la relación entre la traducción y el original, porque solo tratan con la traducción, que para ellos funciona, o debería funcionar, como una obra autónoma. De hecho, para ellos no existe el original.

El segundo comentario es más fundamental. Es una perogrullada que una traducción, por definición, no coincide con el original. Pero no es algo que sea exclusivo de las traducciones. Tampoco los originales coinciden con ellos mismos. El caso es que los textos literarios no se dejan atrapar en interpretaciones o significados definitivos y completos: no por el lector -profesional o no- y ni siquiera por el autor. Al lector se le escapa una parte de cuanto el autor ha querido o pretendido plasmar en la obra. Pero también es verdad lo contrario: el lector extrae del texto significados que ni se le ocurrieron al escritor mientras trabajaba en su libro. Cada lector tiene su propia manera de leer un texto, trátese de una traducción o de un original. Y ni siquiera en este aspecto hay estabilidad: cuando el mismo lector relee el mismo texto su interpretación no tiene por qué ser idéntica a la anterior.

La forma de los textos literarios tampoco se deja encorsetar en una descripción exhaustiva. La narratología ha generado anaqueles de teorías y modelos analíticos, pero ni uno alcanza a captar la forma literaria por completo. Como mucho pueden aspirar a facilitarle al usuario una aproximación sistemática al texto, nada más. Un texto literario es un mundo tan complejo que en última ins- 
tancia solo puede ser representado por este mismo, como es el caso del Imperio en el cuento ultracorto "Del rigor en la ciencia" de Borges:

En aquel Imperio, el Arte de la Cartografía logró tal Perfección que el Mapa de una sola Provincia ocupaba toda una Ciudad, y el Mapa del Imperio, toda una Provincia. Con el tiempo, estos Mapas Desmesurados no satisficieron y los Colegios de Cartógrafos levantaron un Mapa del Imperio, que tenía el Tamaño del Imperio y coincidía puntualmente con él. (Borges 1974)

El relato "Pierre Menard, autor del Quijote" también puede leerse como una alegoría de lo que es la traducción literaria. Pierre Menard -un escritor simbolista del fin de siglo francés y por tanto ni coetáneo de Cervantes ni hermanado con él por el idioma- quiso escribir de nuevo el Quijote, pero no poniéndose en la piel de Cervantes, sino de la siguiente manera: "seguir siendo Pierre Menard y llegar al Quijote, a través de las experiencias de Pierre Menard" (Borges 1985: 129). Así, "[d]edicó sus escrúpulos y vigilias a repetir en un idioma ajeno un libro preexistente" (132). A diferencia de Cervantes, Pierre Menard no disponía de plena libertad al escribir su texto, sino que, igual que un traductor, estaba atado a un texto preexistente. Es más: en el fondo, Menard tradujo el texto de Cervantes. Por otro lado, el resultado de la empresa de Menard en su condición de traducción se caracteriza por algo de lo que, por definición, carece cualquier traducción: es un texto que en cuanto a su forma no se distingue en nada del original y que, por tanto, es una equivalencia integral del original. Pero precisamente gracias a su condición de traducción, el Quijote de Menard -un texto contemporáneo, escrito por un hablante de otro idioma- puede generar otros significados que el original. Por eso el narrador del cuento de Borges no duda en concluir que "[e]l texto de Cervantes y el de Menard son verbalmente idénticos, pero el segundo es casi infinitamente más rico" (131). El Quijote de Menard no sufre ningún cambio formal y por consiguiente es, según el paradigma de la equivalencia, una traducción ideal. Pero teniendo presente los nuevos significados se podría incluso dar un paso más y sostener que la traducción es un texto superior al original.

"Pierre Menard, autor del Quijote" es una ficción sobre una empresa absurda ( $y$, en esencia, fracasada: después de mucho esfuerzo Menard solo logra escribir una pequeña parte del libro). Pero como experimento mental, el texto de Borges aclara con concisión que incluso si en la traducción se mantiene la forma del texto idéntica a la del original -el deseo inalcanzable de la equivalencia que sigue condicionando la mentalidad y la práctica en el mundo de los traductores literarios- el significado del texto no será en absoluto idéntico. Lo que el cuento de Borges sugiere con ello es que una traducción en otro idioma -es decir, una traducción "normal" o "verdadera"- amplía aún más el horizonte semántico del texto. Las traducciones no suponen, por consiguiente, un empobrecimiento del texto, según reza el tópico, sino que también pueden considerarse justamente un enriquecimiento. Así lo recalca y elabora Suzanne Jill Levine en The Subversive Scribe, en que sostiene que "translations uncover subtexts, or underlying meanings" (Levine 1991: 7), un punto de vista que no niega sino que matiza el paradigma de la equivalencia. 


\section{LA TAREA DEL TRADUCTOR}

Un traductor es mucho más que un lector común y corriente, pero tampoco logra medir todo el potencial semántico del texto; al fin y al cabo, su acercamiento será, por definición, individual (Rabinowitz 1998). Sí que puede inventariar la historia de la recepción de la obra que se propone traducir, aunque solo fuera para tener una idea más profunda del texto, aunque esta, por exhaustiva que sea, por definición no puede ser completa. En primer lugar porque es imposible conocer todas las lecturas o interpretaciones realizadas y, en segundo lugar, porque incluso si eso fuera posible no quedarían agotados los posibles significados del texto.

Con ello, la equivalencia no solo es un problema de la traducción, también lo es de la lectura y es, por lo tanto, inherente a todo texto literario, traducido o no. Esta constatación no hace más que añadir complejidades y problemas al proceso de traducir. Porque si el texto original no es completamente asimilable y abarcable por su intricada e incesante movilidad y provisionalidad, entonces ¿a qué se puede asir el traductor? ¿No está su trabajo de antemano condenado al fracaso si el texto que traduce por definición no es el texto completo?

La respuesta sería afirmativa si existiera el lector perfecto. Pero éste no existe, como tampoco existen la lectura y la interpretación perfectas. ¿Por qué, entonces, una traducción perfecta o ideal tendría que ser el criterio para juzgar una traducción de carne y hueso? ¿Por qué la equivalencia completa tendría que ser la norma? Si fuera cierto que es imposible traducir, también lo sería leer. Pero sabemos que la realidad es otra. Leemos y no nos parece ningún problema que todos lo hagamos de otra manera. Por eso no es un problema fundamental que las traducciones varíen respecto al original y también entre ellas. Es, lisa y llanamente, un hecho experiencial.

Y sin embargo, la ambición de los traductores literarios suele ser rendir un texto que sea en la mayor medida posible equivalente al original. El origen de esta idea es que el texto del autor es sagrado -en su esencia, una idea romántica-y que el del traductor debe aproximarse lo más posible a aquel. Pero ¿no es llamativo que escritores bilingües como Borges y Beckett dieran una generosa libertad a los traductores con los que colaboraban? Ambos tenían más conciencia que nadie de que la forma, el significado y el efecto de sus palabras estaban vinculados a la lengua en la que escribían, y que cualquier intento de lograr en otro idioma la misma forma, el mismo significado y el mismo efecto estaba condenado al fracaso. Es inevitable que una traducción literaria difiera en dos aspectos fundamentales de la obra original. En primer lugar, cabe recordar que el estilo de la lengua meta es fundamentalmente diferente al de la lengua fuente, como hemos esbozado más arriba. Y en segundo lugar, conviene destacar que el estilo del traductor por definición no se corresponde con el del autor al que traduce.

La doctrina de la equivalencia solamente presta atención a la primera diferencia. El único problema que habría que resolver serían las diferencias entre los dos idiomas. Al traductor le correspondería la tarea de salvarlas o disimu- 
larlas al máximo. Ello significaría que el traductor sería capaz de representar, cual muñeco de un ventrílocuo, el estilo del escritor y que, por tanto, podría aparcar su propio estilo sin problema alguno. Conviene insistir en que se trata de un malentendido persistente y de proporciones colosales. Todos tenemos nuestro propio estilo. ¿Por qué se manifestaría este en todas las formas en las que usamos la lengua -como cuando escribimos un correo electrónico, cuando hablamos en público, cuando estamos en un bar, o cuando estamos charlando en la cama con nuestra pareja, y hasta cuando tenemos que escribir un tedioso informe- pero no cuando estamos traduciendo? Nuestro estilo no se deja amordazar ni silenciar. Cuando uno echa su propio estilo a la calle vuelve a colarse por la puerta de atrás.

Así se explica que la tarea del traductor es más compleja y abarca más de lo que se suele suponer. Este no solo debe tener un excelente conocimiento de la lengua fuente, formarse una idea muy clara del estilo del escritor y conocer su propio idioma de cabo a rabo, sino que también debería escuchar su propia voz y, de este modo, descubrir y (re)conocer su propio estilo y desarrollar y afinarlo dentro de lo posible (ver Venuti 2013, que destaca el intrincado papel del inconsciente y sus efectos en la traducción). El traductor es, pues, mucho más que un intérprete: es un escritor. No un escritor en el sentido común y corriente de la palabra, como ya habrá quedado claro. Escribe como otro pero, por definición, también como él mismo. Busca sin cesar en su obra-o debería hacerlo- un equilibrio entre la voz del escritor y la propia.

Ahora bien, la voz o el estilo del escritor no es un fenómeno perceptible o descriptible -o mejor dicho: no es exclusivamente un fenómeno perceptible o descriptible- sino una experiencia. Como ya se ha destacado, cada lector interpreta y valora un texto de forma distinta. Sería ingenuo pretender que para un traductor la experiencia de un texto fuera, en esencia, de otra naturaleza que la del lector "normal". Evidentemente, un traductor lee más veces y lee más a fondo el texto original, y, por tanto, lo conoce mejor. Pero la diferencia es de grado, no de esencia. Conoce mejor el texto. Y, asimismo, lo vive más. Por consiguiente, no puede quedar fuera de juego su experiencia del texto. Al contrario: esta es inherente a su labor como traductor.

En su ensayo "Ausencia y memoria en la traducción poética" Javier Marías sostiene que no existe una diferencia fundamental entre traducir literatura y escribirla. Una traducción "no es [...] fotografía, copia. No puede serlo. Un texto, por el hecho de pasar de una lengua a otra, sufre ya una transformación objetiva de tal carácter y magnitud que nunca podrá ser idéntico, ni será el mismo en ambas..." (Marías 2001: 378; cursiva del autor). Traducir es, según el autor madrileño, un acto de creación que se basa en una experiencia específica. No se trata de una experiencia que de un modo $u$ otro esté entrelazada con la realidad (una historia, un suceso, una idea, un estado de ánimo), como suele ser el caso cuando se escribe literatura en el sentido propio de la palabra, sino una experiencia literaria o lingüística. Esta se compone de al menos dos elementos: el modo en que se vive el texto original y la ausencia de ese mismo texto en la propia lengua. Por lo tanto, la traducción no se basa en un texto, porque un texto no es nada 
en sí mismo, según Marías. La traducción se forja a partir del recuerdo de un texto. Eso explicaría también por qué las traducciones varían entre ellas. Ningún recuerdo -ninguna experiencia de un texto- es igual a otro.

El punto de vista holístico de Marías nos salva de caer en la tentación de enfangarnos con las discusiones bizantinas que caracterizan muchos de los debates sobre la calidad de las traducciones y nos obliga a reflexionar sobre lo que, en el fondo, es la traducción literaria. Surgen las preguntas: ¿Qué es lo que implica exactamente la experiencia o el recuerdo del original? ¿Y cómo podría un traductor plasmar esta experiencia en su lengua materna?

El traductor podría ofrecer la respuesta a las primeras pregunta en su condición de lector. Es en esa calidad que va construyendo un vínculo con el texto que va a traducir, que obviamente es mucho más intenso que en el caso de un lector común. Y quizá más que con el texto, el traductor se relacione con su escritor. Mejor dicho: con la representación o la idea que él o ella se construye de la persona que ha creado el texto, basadas principalmente en los textos del autor que haya leído, pero también en lo que además sepa de él, haya leído sobre él o, a lo mejor, haya vivido con él.

Así, el escritor, su mundo y su estilo van tomando posesión, con cada vez más fuerza, de la cabeza del traductor, y aquel no solo está presente cuando éste lo lee, sino también cuando no lo lee. El texto y el escritor van amalgamándose hasta convertirse en recuerdo. Este recuerdo -que también actúa como una especie de conciencia que resguarda al traductor de apropiarse en exceso del texto y usurparlo- varía según cada traductor, pero no es arbitrario. Los escritores se distinguen, también como representación o recuerdo. El escritor elige un tema así como la forma que le parezca más ajustada al mismo, pero ya solamente en la elección de la forma y desde luego en la escritura está condicionado por su propia voz. Bien es cierto que es un tópico que los escritores siempre escriben el mismo libro, pero no por ello es menos cierto, en el fondo. Que Borges, por poner un ejemplo, jamás escribiera novelas no fue una decisión premeditada, como él mismo gustaba hacer creer. El caso es que era incapaz de escribirlas.

Aunque el traductor sepa que la voz del escritor no es un fenómeno objetivo sino una experiencia, cree no obstante que lo que él se ha grabado en la cabeza es la voz de este. O para acudir a la atinada expresión del malogrado escritor holandés Frans Kellendonk (1992): un traductor debería fingir con sinceridad la voz del escritor con su propia voz. Porque ¿cuál sería la alternativa? Un probado procedimiento didáctico -analizar primero el texto a fondo y traducirlo sólo después- permitiría hacer un inventario del texto lo más detallado posible: de la sintaxis, del léxico, de la prosodia, de los procedimientos retóricos, de la intertextualidad, de la recepción... Evidentemente, en sí no sería mala idea que uno primero se haga una idea de las características del original, pero sí conlleva un peligro que no cabe subestimar: cuanto más detallada la descripción, más pesado será el yugo que tenga que soportar el traductor. Antes de que siquiera haya traducido una sola palabra, ya va agachado bajo una batería de características formales (pero ¿qué significan estas, en realidad? y ¿qué relación guardan?) y las posibilidades semánticas del texto (en el fondo inagotables). Todo traductor 
sabe que jamás conseguirá del todo embutir en su traducción todas las características formales con todo su potencial semántico. Sabe que tiene que elegir y, por consiguiente, excluir. La mejor pauta es en ese caso la voz del escritor que se ha anidado como un recuerdo en su cabeza. Un recuerdo idiosincrático. Por razón de ello, el traductor traduce a su escritor.

\section{3. ¿ESCRIBIR COMO OTRO?}

Viene al caso aquí recalcar que la forma de vivir el texto original es, en el fondo, una experiencia musical. Los textos literarios nos instalan en un determinado estado de ánimo. Leer es escuchar. O como lo formuló Stevenson en "On Some Technical Elements of Style in Literature": "Literature is written by and for two senses: a sort of internal ear, quick to perceive 'unheard melodies'; and the eye, which directs the pen and deciphers the printed phrase" (Stevenson 1905). No me consta que ya se haya investigado, pero me parece muy probable que los textos bien escritos -o, mejor dicho, bien compuestos- tengan, en el fondo, el mismo efecto en nuestro cerebro que la música (caso para el cual esto ya ha quedado demostrado): producen endorfinas, sustancia que no solo mitiga el dolor, sino que también nos pone contentos o incluso nos hace felices. Esto explicaría por qué no nos cansamos nunca de determinados escritores, al igual que por qué queremos volver a escuchar una y otra vez a ciertos músicos o compositores. Conocemos todas sus obras, las tenemos grabadas en nuestra memoria y aun así las seguimos leyendo y releyendo. Eso tiene que ver, evidentemente, con las posibilidades en principio inagotables de las mismas. Pero hay, además, otra razón, y tal vez mucho más importante, que es la musicalidad adictiva de sus obras.

La lectura de obras literarias no es solamente una experiencia intelectual, y quizá ni siquiera lo sea en primer lugar, sino también, y tal vez antes que nada, una experiencia física. Probablemente, el síntoma más evidente de ello es que tendemos a leer a paso de tortuga a los escritores que más nos gustan y solemos volver a sus textos. De este modo y tal como ocurre con la música, procuramos recobrar el estado de felicidad o, en los casos más sublimes, la epifanía. Es cierto que en primera instancia el significado complejo nos exige mucha atención, pero en el fondo es la experiencia corporal la que vivimos con más intensidad. Por eso es preciso calibrar el conocido argumento de que la literatura importa porque nos desazona, nos incomoda, nos saca de nuestra zona de confort. No valoramos los textos literarios en primer lugar porque nos produzcan inquietud, sino porque nos generan un encantamiento. Los escritores lo consiguen con su estilo, mientras que los traductores lo consiguen no solo con el estilo del escritor sino también con el propio. Por esta razón, el traductor no es un escritor únicamente a medias, que tiene o debería tener el talento técnico del escritor "de verdad", aunque sin su poder de imaginación. No, el traductor es un escritor por partida doble. Escribe como otro y además como él mismo.

Reivindicando el intricado arte del traductor, Peter Bush ha calificado la traducción como "the boldest act of writing" (2006: 24). De hecho, los traducto- 
res constituyen una categoría singular de escritores, y las traducciones, a su vez, una categoría singular de textos literarios. En este contexto vale la pena recordar lo que Borges asevera en el magnífico ensayo "Las versiones homéricas": para aproximarse al misterio de la literatura son más adecuadas las traducciones que los originales. Precisamente debido a las diferencias entre ellas y por la imposibilidad fundamental de coincidir con los textos originales, las traducciones hacen relucir en todo su esplendor el rasgo definitorio de los textos literarios: su provisionalidad, su movilidad. Una traducción es una foto de un momento determinado, una versión. Más que los originales, las traducciones dejan claro algo que un texto puede leerse e interpretarse de muchas maneras. En este sentido hasta podría considerarse la traducción como superior al original: una traducción no solo expresa uno de los posibles significados del texto (aunque esta por sí sola también admita, a su vez, varias interpretaciones más), sino que asimismo constituye una expresión de la propia movilidad del texto literario.

Es una evidencia que las traducciones no coinciden con el original y que, al mismo tiempo, tampoco son ajenas a este. Son autónomas pero no independientes. Esta disyuntiva entre autonomía y dependencia se parece a la de la literatura misma. Una novela no es una equivalencia de la realidad, sino que representa un mundo autónomo dependiente de (o correlacionado con) el mundo real o auténtico. "En la narración", según apunta el filósofo holandés Ger Groot en Vergeten te bestaan [Olvidarse de existir], "todo se centra en borrar la apariencia de lo ficticio y en presentar el mundo que se describe como verídico" (Groot 2010: 11; la traducción es nuestra). Y, podría añadirse: en presentar el mundo que se describe como real. En el fondo una traducción hace lo mismo. Una traducción no es "real" o "auténtica", no es el original, sino una versión del original. Pero esta versión sí pretende parecer auténtica y real. Y lo es, de hecho. Lo es en la experiencia del lector. Aunque este sepa que está leyendo una traducción -no el texto del escritor, sino el del traductor- sí tiene la impresión de estar leyendo la obra del autor original, en la misma medida en que tiene la impresión de estar leyendo sobre personas reales en un mundo real, aunque sepa que se trata de una novela, y aunque la etiqueta de "novela" que suelen exhibir los libros en su portada y en su primera página impresa se lo vuelva a recordar una vez más.

Igual que un escritor miente la realidad, así el traductor miente el original. Podría decirse por eso que indudablemente sí existe la equivalencia entre el original y la traducción, al igual que existe la equivalencia entre el mundo real y los mundos que vivimos cuando leemos una novela: existe como una ilusión. Una ilusión que compartimos. O sea, una ilusión social. Sobre esa base podría decirse incluso que las traducciones son el género literario por excelencia. Fingen por duplicado: fingen que son el original y que, al igual que el original, tratan sobre la realidad.

Por todas estas razones es coherente concluir que una buena traducción no es inferior al original sino que se yuxtapone a este. Por eso no debería faltar el nombre del traductor en la portada del libro. $Y$ en la contraportada, o en algún otro lugar del libro, no solo debería aparecer información sobre el autor, sino también un breve resumen de los trabajos del traductor, pues si es cierto 
que una traducción literaria no existiría sin el texto original, también lo es que la traducción literaria no existiría sin el traductor. Es más: la traducción literaria depende de su autor, es decir, el traductor: un escritor bifronte cuya patria es la zona fantasma donde se encuentran y se entretejen el estilo del autor y el estilo del traductor.

\section{OBRAS CITADAS}

Bellos, David (2011): Is That a Fish in Your Ear? Translation and the Meaning of Everything. Londres, Particular Books (Penguin).

Borges, Jorge Luis (1974): "Del rigor en la ciencia". En: Obras Completas 1923-1972. 14a edición. Buenos Aires, Emecé Editores, p. 847.

— (1985): "Pierre Menard, autor del Quijote". En: Prosa completa 2. Barcelona, Bruguera, pp. $125-133$.

Boroditsky, Lera; Schmidt, Lauren A.; y Phillips, Webb (2003): "Sex, Syntax, and Semantics". En: Dedre Gentner y Susan Goldin-Meadow (eds.): Language in Mind. Advances in the Study of Language and Thought. Cambridge (Massachusetts) / Londres, The MIT Press, pp. 61-79.

Durastanti, Sylvia (2002): Éloge de la trahison: notes du traducteur. París / Nueva York, Le Passage.

Groot, Ger (2010): Vergeten te bestaan. Echte fictie en het fictieve ik. Nimega, Vantilt.

Kellendonk, Frans (1992): "Idolen". En: Verzameld werk. Ámsterdam, Meulenhoff.

Levine, Suzanne Jill (1991): The Subversive Scribe. Translating Latin American Fiction. Saint Paul, Graywolf Press.

Marías, Javier (2001): "Ausencia y memoria en la traducción poética". En: Literatura y fantasma. Edición ampliada. Madrid, Alfaguara, pp. 371-382.

Mayhew, Jonathan (2009): Apocryphal Lorca. Translation, Parody, Kitsch. Chicago/Londres, The University of Chicago Press.

Mendoza, Eduardo (2002): "La traducción y sus descontentos". Barcelona, Universitat Pompeu Fabra. Facultat de Traducció i Interpretació. Disponible en <http://http//www. upf.edu/factii/activitatsfti/llicons/mendoza.html> [última visita: 18.01.2016].

Rabinowitz, Peter. J. (1998): Before Reading: Narrative Conventions and the Politics of Interpretation. Columbus, Ohio State University Press.

Schopenhauer, Arthur (1996): "Sobre lengua y palabras". Trad. Hans Christian Hagedorn. En: Dámaso López García (ed.): Teorías de la traducción: antología de textos. Cuenca, Universidad de Castilla-La Mancha, pp. 197-202.

Stevenson, Robert Louis (1905): "On Some Technical Elements of Style in Literature". En: The Art of Writing. Disponible en <http://classiclit.about.com/library/bl-etexts/rlstevenson/bl-rlst-wri-1.htm> [última visita: 18.01.2016].

Venuti, Lawrence (2013): "The Difference that Translation Makes. The Translator's Unconscious". En: Translation Changes Everything. Theory and Practice. Londres / Nueva York, Routledge, pp. 32-56. 